\title{
Distinguishing natural from anthropogenic sources of acid extractable organics in groundwater near oil sands tailings ponds
}

\author{
Jason M. E. Ahad ${ }^{1 *}$, Hooshang Pakdel ${ }^{2}$, Paul R. Gammon ${ }^{3}$, Bernhard Mayer ${ }^{4}$, Martine M. \\ Savard $^{1}$, Kerry M. Peru ${ }^{5}$ and John V. Headley ${ }^{5}$
}

${ }^{1}$ Geological Survey of Canada, Natural Resources Canada, Québec City, QC, G1K 9A9, Canada, jason.ahad@,canada.ca (*corresponding author)

${ }^{2}$ INRS Eau Terre Environnement, Québec City, QC, G1K 9A9, Canada

${ }^{3}$ Geological Survey of Canada, Natural Resources Canada, Ottawa, ON, K1A 0E8, Canada

${ }^{4}$ Department of Geoscience, University of Calgary, Calgary, AB, T2N 1N4, Canada

${ }^{5}$ Water Science and Technology Directorate, Environment and Climate Change Canada, Saskatoon, SK, S7N 3H5, Canada

Number of pages: 10

Number of tables: 3

Number of figures: 2 
Table S1: Summary of samples collected and analyzed (NA = not available), including sample type, date collected, electrical conductivity $(\mu \mathrm{S} / \mathrm{cm}), \mathrm{pH}$, temperature $\left({ }^{\circ} \mathrm{C}\right)$, dissolved oxygen $(\mathrm{DO} ; \mathrm{mg} / \mathrm{L})$, and concentrations $(\mathrm{mg} / \mathrm{L}), \delta^{13} \mathrm{C}_{\mathrm{pyr}}$ and $\delta^{34} \mathrm{~S}$ values $(\% 0)$ of acid extractable organics (AEOs).

\begin{tabular}{|c|c|c|c|c|c|c|c|c|c|}
\hline Sample & Sample Type & Date collected & $\begin{array}{c}E C \\
(\mu \mathrm{S} / \mathrm{cm})\end{array}$ & $\mathrm{pH}$ & $\begin{array}{c}\text { Temp } \\
\left({ }^{\circ} \mathrm{C}\right)\end{array}$ & $\begin{array}{c}\mathrm{DO} \\
\text { (mg/L) }\end{array}$ & $\begin{array}{l}\text { AEOs } \\
\text { (mg/L) }\end{array}$ & $\begin{array}{c}\delta^{13} C_{\text {pyr of }} \\
\text { AEOS (\%o) }\end{array}$ & $\begin{array}{c}\delta^{34} S \text { of } \\
\text { AEOs (\%o) }\end{array}$ \\
\hline TP1-2014 & Tailings pond (OSPW) & $11-2014$ & 2970 & 8.3 & NA & NA & 81.0 & -21.1 & 7.1 \\
\hline TP2-2015 & Tailings pond (OSPW) & $22-10-2015$ & 2410 & 7.0 & NA & NA & 72.1 & -20.6 & 6.6 \\
\hline TP2-2016 & Tailings pond (OSPW) & $27-09-2016$ & 1699 & 8.3 & 12.8 & 4.0 & 65.5 & -20.2 & 6.4 \\
\hline TP2-2017 & Tailings pond (OSPW) & $13-09-2017$ & 1426 & 8.1 & 13.8 & 2.5 & NA & -20.8 & 7.0 \\
\hline SP-2015 & Seep-pipe & $22-10-2015$ & 2065 & 6.9 & 12.4 & 4.1 & 50.4 & -23.1 & 6.4 \\
\hline SP-2016 & Seep-pipe & $27-09-2016$ & 2731 & 6.7 & 10.3 & 0.3 & 41.2 & -23.9 & 4.7 \\
\hline SP-2017 & Seep-pipe & $12-09-2017$ & 1510 & 7.1 & 11.3 & 8.1 & NA & -20.2 & 7.3 \\
\hline MFGW-1-2014 & McMurray Formation GW & $21-10-2014$ & 1753 & 7.0 & NA & NA & 20.6 & -21.5 & 10.2 \\
\hline MFGW-2-2014 & McMurray Formation GW & $19-10-2014$ & 1282 & 7.4 & 8.0 & 0.9 & 5.8 & -21.9 & 8.8 \\
\hline MFGW-1-2015 & McMurray Formation GW & $22-10-2015$ & 2540 & 7.2 & NA & NA & 19.4 & -22.6 & 7.5 \\
\hline MR-UP-2015 & Muskeg River (non-bitumen) & $18-10-2015$ & 235 & 7.4 & 6.1 & 3.9 & 5.4 & -28.6 & 5.0 \\
\hline MR-UP-2016 & Muskeg River (non-bitumen) & 08-10-2016 & 275 & 7.3 & 2.0 & 6.8 & 1.5 & -27.4 & 5.3 \\
\hline MR-UP-2017 & Muskeg River (non-bitumen) & $13-09-2017$ & 353 & 7.4 & 12.7 & 3.5 & NA & -28.3 & 4.9 \\
\hline PGW-2014 & Pleistocene GW (non-bitumen) & $21-10-2014$ & 874 & 6.9 & NA & NA & 5.2 & -27.3 & 7.0 \\
\hline PGW-2015 & Pleistocene GW (non-bitumen) & $22-10-2015$ & 1020 & 7.3 & NA & NA & 7.1 & -27.9 & 6.9 \\
\hline MR-01-2014 & Muskeg River & $20-10-2014$ & 332 & 8.2 & 4.6 & 12.7 & 4.5 & -28.2 & 6.2 \\
\hline MR-J6N-2014 & Muskeg River & 23-10-2014 & 388 & 7.6 & 5.0 & 9.4 & 4.3 & -28.1 & 4.9 \\
\hline MR-01-2015 & Muskeg River & $17-10-2015$ & 246 & 8.4 & 5.2 & 12.3 & 7.8 & -27.9 & NA \\
\hline MR-02-2015 & Muskeg River & 22-10-2015 & 226 & 8.0 & 5.9 & 9.7 & 7.6 & -27.8 & NA \\
\hline MR-02-2016 & Muskeg River & 28-09-2016 & 285 & 7.7 & 8.2 & 6.7 & 1.8 & -28.0 & 4.4 \\
\hline MR-1023-2016 & Muskeg River & 07-10-2016 & 264 & 8.0 & 4.1 & 9.1 & 1.8 & -27.4 & 4.1 \\
\hline MR-01-2016 & Muskeg River & $29-09-2016$ & 301 & 8.5 & 8.6 & 8.9 & 1.8 & -27.4 & 5.6 \\
\hline MR-01-2017 & Muskeg River & $11-09-2017$ & 412 & 8.3 & 14.0 & 8.4 & NA & -27.7 & 5.5 \\
\hline MR-02-2017 & Muskeg River & $13-09-2017$ & 374 & 8.2 & 13.2 & 7.9 & NA & -27.8 & 4.8 \\
\hline MR-04-2017 & Muskeg River & 01-09-2017 & 395 & 7.9 & 17.8 & 8.4 & NA & -28.0 & 4.6 \\
\hline SW-01-2014 & Wetland/stream (surface water) & $23-10-2014$ & 375 & 7.8 & 6.9 & 8.4 & 2.5 & -27.4 & 8.1 \\
\hline
\end{tabular}




\begin{tabular}{|c|c|c|c|c|c|c|c|c|c|}
\hline SW-02-2014 & Wetland/stream (surface water) & 23-10-2014 & 505 & 7.4 & 7.4 & 6.6 & 1.8 & -27.9 & 5.8 \\
\hline JPC-01-2016 & Wetland/stream (surface water) & $28-09-2016$ & 263 & 7.7 & 7.7 & 7.6 & 1.8 & -28.3 & 3.0 \\
\hline DP-25S-2016 & Wetland/stream (surface water) & 07-10-2016 & 283 & 7.3 & 3.1 & 7.6 & 2.0 & -26.4 & 6.9 \\
\hline AR-5W-2014 & Athabasca River & $10-2014$ & 306 & 7.8 & NA & NA & 1.5 & -27.3 & 0.3 \\
\hline AR-20e-2014 & Athabasca River & $10-2014$ & 267 & 7.7 & NA & NA & 1.5 & -27.9 & NA \\
\hline AR-15W-2014 & Athabasca River & $10-2014$ & 297 & 7.8 & NA & NA & 1.5 & -27.4 & NA \\
\hline AR-18W-2014 & Athabasca River & $10-2014$ & 277 & 7.8 & NA & NA & 1.0 & -27.5 & NA \\
\hline AR-00-2015 & Athabasca River & $17-10-2015$ & 246 & 8.4 & 6.4 & 12.6 & 1.4 & -27.3 & NA \\
\hline AR-02-2015 & Athabasca River & $22-10-2015$ & 177 & 8.4 & 7.2 & 11.8 & 1.3 & -26.9 & NA \\
\hline AR-04-2015 & Athabasca River & $17-10-2015$ & 237 & 8.4 & 6.8 & 11.0 & 1.6 & -26.5 & NA \\
\hline AR-00-2016 & Athabasca River & $05-10-2016$ & 302 & 8.3 & 4.9 & 9.5 & 1.4 & -28.3 & 1.8 \\
\hline AR-00-2017 & Athabasca River & 02-09-2017 & 265 & 8.4 & 18.1 & 8.5 & NA & -27.9 & NA \\
\hline GW1-1-2014 & Site 1 monitoring well GW & $17-10-2014$ & 1050 & 6.7 & 5.0 & 0.8 & 2.7 & -27.3 & 5.3 \\
\hline GW1-2-2014 & Site 1 monitoring well GW & 23-10-2014 & 1072 & 7.0 & 6.7 & 0.3 & 7.2 & -25.4 & 7.3 \\
\hline GW1-3-2014 & Site 1 monitoring well GW & $16-10-2014$ & 1089 & 7.2 & 9.0 & 0.5 & 6.5 & -26.0 & 8.2 \\
\hline GW1-4-2014 & Site 1 monitoring well GW & $17-10-2014$ & 1005 & 7.0 & 5.6 & 0.4 & 5.2 & -26.3 & 8.2 \\
\hline GW1-5-2014 & Site 1 monitoring well GW & $16-10-2014$ & 892 & 7.2 & 8.1 & 0.7 & 6.1 & -26.0 & 7.0 \\
\hline GW1-6-2014 & Site 1 monitoring well GW & $18-10-2014$ & 596 & 6.8 & 8.0 & 0.0 & 8.5 & -26.4 & 6.6 \\
\hline GW1-7-2014 & Site 1 monitoring well GW & $19-10-2014$ & 1106 & 7.0 & 7.0 & 0.0 & 5.5 & -25.8 & 7.6 \\
\hline GW1-8-2014 & Site 1 monitoring well GW & $18-10-2014$ & 683 & 7.1 & 8.7 & 1.0 & 7.2 & -25.5 & 8.4 \\
\hline GW1-9-2014 & Site 1 monitoring well GW & $18-10-2014$ & 685 & 7.2 & 6.3 & 0.1 & 2.8 & -26.1 & 8.2 \\
\hline GW2-1-2015 & Site 2 monitoring well GW & $19-10-2015$ & 765 & 6.9 & 6.1 & 3.9 & 11.2 & -26.0 & 6.0 \\
\hline GW2-2-2015 & Site 2 monitoring well GW & $21-10-2015$ & 1239 & 7.0 & 5.4 & 0.7 & 26 & -21.9 & 6.7 \\
\hline GW2-3-2015 & Site 2 monitoring well GW & $20-10-2015$ & 1518 & 6.7 & 8.9 & 7.1 & 58.9 & -26.8 & 5.9 \\
\hline GW2-4-2015 & Site 2 monitoring well GW & $20-10-2015$ & 838 & 6.9 & 7.0 & 0.4 & 10.1 & -25.9 & 5.2 \\
\hline GW2-5-2015 & Site 2 monitoring well GW & $21-10-2015$ & 915 & 6.6 & 9.7 & 4.3 & 12.9 & -24.7 & 7.4 \\
\hline GW2-6-2015 & Site 2 monitoring well GW & $22-10-2015$ & 1300 & 6.6 & 9.3 & 0.9 & 15.2 & -24.8 & 7.0 \\
\hline GW2-7-2015 & Site 2 monitoring well GW & $22-10-2015$ & 1247 & 7.1 & 8.1 & 0.4 & 12.8 & -26.7 & 6.3 \\
\hline GW2-8-2015 & Site 2 monitoring well GW & $21-10-2015$ & 1065 & 6.9 & 8.9 & 0.5 & 7.3 & -27.5 & 6.7 \\
\hline DP-25-2016 & Site 2 monitoring well GW & $07-10-2016$ & 1043 & 6.4 & 6.8 & 0.7 & 5.5 & -26.7 & 5.4 \\
\hline DP-17-2016 & Site 2 monitoring well GW & $09-10-2016$ & 582 & 6.4 & 5.6 & 0.2 & 7.7 & -26.8 & 5.5 \\
\hline DP-24-2016 & Site 2 monitoring well GW & $06-10-2016$ & 1327 & 6.7 & 5.1 & 1.3 & 8.2 & -26.8 & 6.5 \\
\hline GW2-9-2016 & Site 2 monitoring well GW & $08-10-2016$ & 1406 & 6.4 & 4.6 & 2.6 & 12.3 & -27.0 & 5.9 \\
\hline DP-04-2016 & Site 2 monitoring well GW & 03-10-2016 & 1493 & 6.9 & 7.0 & 0.8 & 8.1 & -26.3 & 5.6 \\
\hline GW2-3-2016 & Site 2 monitoring well GW & $02-10-2016$ & 2217 & 6.6 & 11.5 & 2.4 & 24.9 & -27.2 & 6.9 \\
\hline
\end{tabular}




\begin{tabular}{|c|c|c|c|c|c|c|c|c|c|}
\hline GW2-2-2016 & Site 2 monitoring well GW & $27-09-2016$ & 1837 & 7.1 & 6.6 & 0.2 & 24.7 & -23.1 & 6.6 \\
\hline DP-02-2017 & Site 2 monitoring well GW & 07-09-2017 & 924 & 7.3 & 10.7 & 0.9 & NA & -20.5 & 7.0 \\
\hline DP-10-2017 & Site 2 monitoring well GW & 06-09-2017 & 889 & 7.0 & 13.5 & 2.6 & NA & -22.9 & 8.0 \\
\hline DP-21-2017 & Site 2 monitoring well GW & 09-09-2017 & 576 & 6.7 & 7.9 & 0.6 & NA & -25.8 & 7.5 \\
\hline DP-15-2017 & Site 2 monitoring well GW & 08-09-2017 & 1082 & 10.5 & 7.2 & 0.2 & NA & -25.0 & 6.6 \\
\hline DP-24-2017 & Site 2 monitoring well GW & 02-09-2017 & 1252 & 6.7 & 8.5 & 1.0 & NA & -24.6 & 6.5 \\
\hline GW2-7-2017 & Site 2 monitoring well GW & $12-09-2017$ & 1073 & 7.1 & 8.7 & 0.3 & NA & -25.1 & 6.2 \\
\hline GW2-9-2017 & Site 2 monitoring well GW & $10-09-2017$ & 1135 & 6.4 & 10.7 & 2.5 & NA & -25.9 & 6.5 \\
\hline GW2-3-2017 & Site 2 monitoring well GW & 14-09-2017 & 718 & 6.5 & 10.7 & 2.9 & NA & -27.2 & 6.2 \\
\hline
\end{tabular}


Table S2. The percentage (\%) of dominant species classes in acid extractable organics (AEOs) determined by high resolution Orbitrap mass spectrometry. See Table S1 for further sample information.

\begin{tabular}{|c|c|c|c|c|c|c|c|c|c|c|c|c|c|c|c|c|}
\hline Sample & 0 & $\mathrm{O} 2$ & 03 & 04 & 05 & 06 & 07 & 08 & 09 & 010 & $\mathrm{Nx}$ & NxSx & NxOx & NxOxSx & OxSx & Sx \\
\hline TP1-2014 & 0.0 & 58.7 & 7.9 & 20.1 & 0.3 & 0.0 & 0.0 & 0.0 & 0.0 & 0.0 & 0.0 & 0.0 & 0.0 & 0.1 & 12.8 & 0.2 \\
\hline TP2-2015 & 0.0 & 60.1 & 9.1 & 14.3 & 0.5 & 0.0 & 0.0 & 0.0 & 0.0 & 0.0 & 0.0 & 0.0 & 0.0 & 0.1 & 15.8 & 0.0 \\
\hline TP2-2016 & 0.2 & 67.9 & 5.2 & 11.2 & 0.0 & 0.0 & 0.0 & 0.0 & 0.0 & 0.0 & 1.8 & 0.0 & 0.0 & 0.3 & 13.0 & 0.5 \\
\hline TP2-2017 & 0.1 & 74.4 & 5.8 & 10.8 & 0.0 & 0.0 & 0.0 & 0.0 & 0.0 & 0.0 & 0.9 & 0.0 & 0.1 & 0.0 & 8.0 & 0.0 \\
\hline SP-2015 & 0.0 & 49.3 & 9.0 & 21.1 & 1.9 & 0.4 & 0.0 & 0.0 & 0.0 & 0.0 & 0.0 & 0.0 & 0.0 & 0.1 & 17.9 & 0.1 \\
\hline SP-2016 & 0.3 & 51.7 & 11.6 & 19.5 & 1.0 & 0.1 & 0.0 & 0.0 & 0.0 & 0.0 & 1.7 & 0.0 & 0.1 & 0.4 & 11.4 & 2.3 \\
\hline SP-2017 & 0.2 & 78.0 & 2.7 & 7.1 & 0.0 & 0.0 & 0.0 & 0.0 & 0.0 & 0.0 & 0.5 & 0.0 & 0.2 & 0.0 & 11.0 & 0.3 \\
\hline MFGW-1-2014 & 0.0 & 57.5 & 2.5 & 17.1 & 0.1 & 0.0 & 0.0 & 0.0 & 0.0 & 0.0 & 0.0 & 0.0 & 0.0 & 0.4 & 21.9 & 0.3 \\
\hline MFGW-2-2014 & 0.0 & 56.9 & 2.2 & 12.7 & 0.2 & 0.1 & 0.0 & 0.0 & 0.0 & 0.0 & 0.0 & 0.0 & 0.2 & 0.1 & 26.7 & 0.0 \\
\hline MFGW-1-2015 & 0.0 & 61.1 & 3.0 & 15.2 & 0.6 & 0.7 & 0.0 & 0.0 & 0.0 & 0.0 & 0.0 & 0.0 & 0.1 & 0.3 & 18.6 & 0.3 \\
\hline MR-UP-2015 & 0.1 & 1.5 & 2.3 & 10.5 & 15.0 & 19.5 & 16.9 & 13.6 & 8.0 & 3.9 & 1.4 & 0.7 & 1.3 & 1.5 & 2.5 & 0.1 \\
\hline MR-UP-2016 & 0.9 & 5.9 & 4.2 & 9.4 & 15.8 & 18.6 & 14.0 & 9.5 & 5.9 & 2.7 & 1.9 & 0.5 & 1.3 & 1.3 & 7.9 & 0.4 \\
\hline MR-UP-2017 & 1.5 & 7.0 & 7.2 & 13.9 & 17.1 & 14.0 & 6.6 & 4.4 & 2.4 & 1.0 & 0.7 & 0.7 & 2.9 & 1.1 & 16.1 & 3.4 \\
\hline PGW-2014 & 0.2 & 23.4 & 5.1 & 16.1 & 10.1 & 11.8 & 8.5 & 6.7 & 3.7 & 1.6 & 0.8 & 0.2 & 0.9 & 0.7 & 9.2 & 0.2 \\
\hline PGW-2015 & 0.1 & 28.5 & 5.8 & 16.1 & 9.5 & 11.0 & 8.8 & 5.8 & 3.1 & 1.1 & 0.4 & 0.1 & 0.1 & 0.0 & 9.1 & 0.1 \\
\hline MR-01-2014 & 0.5 & 2.6 & 2.8 & 9.8 & 13.3 & 15.0 & 13.8 & 10.8 & 6.9 & 3.3 & 2.3 & 1.3 & 3.7 & 5.0 & 3.5 & 0.6 \\
\hline MR-J6N-2014 & 0.8 & 3.0 & 2.5 & 9.4 & 12.0 & 15.6 & 14.1 & 10.4 & 6.2 & 3.4 & 2.4 & 1.4 & 4.3 & 5.8 & 3.9 & 0.9 \\
\hline MR-01-2015 & 0.2 & 5.9 & 4.2 & 12.1 & 14.2 & 16.9 & 13.6 & 9.0 & 5.9 & 2.6 & 1.2 & 0.8 & 2.3 & 2.7 & 5.3 & 0.3 \\
\hline MR-02-2015 & 0.4 & 5.5 & 4.2 & 13.2 & 14.1 & 15.2 & 11.6 & 9.7 & 5.7 & 2.4 & 1.5 & 1.2 & 2.9 & 3.3 & 5.7 & 0.3 \\
\hline MR-02-2016 & 0.9 & 4.9 & 5.0 & 12.0 & 17.7 & 18.8 & 12.9 & 8.6 & 5.0 & 2.1 & 1.4 & 0.6 & 0.9 & 1.1 & 7.6 & 0.5 \\
\hline MR-1023-2016 & 0.8 & 4.4 & 4.8 & 12.0 & 17.7 & 18.2 & 12.2 & 8.1 & 4.7 & 2.0 & 1.4 & 0.8 & 1.2 & 1.4 & 9.7 & 0.7 \\
\hline MR-01-2016 & 1.0 & 8.9 & 5.9 & 12.4 & 16.8 & 17.3 & 11.8 & 8.0 & 4.5 & 1.9 & 1.3 & 0.5 & 0.8 & 1.3 & 7.1 & 0.6 \\
\hline MR-01-2017 & 1.2 & 14.5 & 9.9 & 22.8 & 13.3 & 9.7 & 3.9 & 2.7 & 1.8 & 0.7 & 0.9 & 0.4 & 1.3 & 0.6 & 13.9 & 2.4 \\
\hline MR-02-2017 & 1.2 & 13.8 & 9.7 & 21.7 & 14.1 & 9.7 & 4.0 & 2.8 & 1.5 & 0.7 & 0.8 & 0.4 & 1.4 & 0.6 & 14.7 & 2.7 \\
\hline MR-04-2017 & 1.0 & 19.0 & 10.3 & 23.7 & 11.6 & 7.5 & 3.5 & 2.4 & 1.2 & 0.3 & 0.8 & 0.2 & 1.3 & 0.5 & 14.8 & 1.8 \\
\hline SW-01-2014 & 0.3 & 7.4 & 3.9 & 13.1 & 11.8 & 13.0 & 12.7 & 8.8 & 6.3 & 3.5 & 2.1 & 1.1 & 3.5 & 3.8 & 5.2 & 0.6 \\
\hline SW-02-2014 & 0.4 & 5.0 & 4.1 & 13.6 & 11.2 & 13.6 & 13.3 & 10.5 & 7.3 & 3.7 & 2.0 & 0.8 & 2.9 & 2.9 & 5.4 & 0.0 \\
\hline JPC-01-2016 & 0.7 & 3.7 & 3.8 & 8.7 & 16.0 & 18.9 & 14.2 & 9.1 & 5.5 & 2.5 & 1.7 & 0.9 & 1.4 & 2.0 & 10.0 & 0.9 \\
\hline
\end{tabular}




\begin{tabular}{|c|c|c|c|c|c|c|c|c|c|c|c|c|c|c|c|c|}
\hline DP-25S-2016 & 0.9 & 5.2 & 8.0 & 19.5 & 17.4 & 14.9 & 9.5 & 6.2 & 3.6 & 1.4 & 1.2 & 0.3 & 0.3 & 0.7 & 10.3 & 0.5 \\
\hline AR-5W-2014 & 0.6 & 0.8 & 1.7 & 5.2 & 10.7 & 14.4 & 15.0 & 11.8 & 8.8 & 4.5 & 3.9 & 1.5 & 6.2 & 5.7 & 2.7 & 0.4 \\
\hline AR-20e-2014 & 0.5 & 1.5 & 1.5 & 6.8 & 13.2 & 17.7 & 17.5 & 13.0 & 8.5 & 4.8 & 2.6 & 0.9 & 3.2 & 3.2 & 1.6 & 0.6 \\
\hline AR-15W-2014 & 0.7 & 1.2 & 1.4 & 4.1 & 8.6 & 15.1 & 17.3 & 14.7 & 10.1 & 6.2 & 4.1 & 1.2 & 4.6 & 4.2 & 2.6 & 1.0 \\
\hline AR-18W-2014 & 0.3 & 2.6 & 1.7 & 5.1 & 11.8 & 16.7 & 17.8 & 13.6 & 9.3 & 5.7 & 3.3 & 0.9 & 3.6 & 2.9 & 1.9 & 0.8 \\
\hline AR-00-2015 & 0.3 & 3.1 & 2.8 & 9.1 & 14.7 & 19.3 & 16.4 & 11.8 & 7.3 & 3.7 & 1.7 & 0.7 & 2.1 & 2.1 & 2.3 & 0.2 \\
\hline AR-02-2015 & 0.3 & 3.2 & 3.2 & 10.2 & 14.9 & 17.3 & 15.4 & 11.1 & 6.6 & 3.5 & 1.6 & 0.7 & 2.8 & 2.8 & 2.3 & 0.2 \\
\hline AR-04-2015 & 0.2 & 2.5 & 2.9 & 9.5 & 14.5 & 18.0 & 15.8 & 12.2 & 7.2 & 3.3 & 1.8 & 0.8 & 2.2 & 2.7 & 3.4 & 0.1 \\
\hline AR-00-2016 & 1.0 & 5.1 & 4.1 & 9.5 & 17.2 & 20.1 & 14.5 & 9.3 & 5.7 & 2.5 & 1.5 & 0.3 & 1.0 & 1.2 & 6.3 & 0.6 \\
\hline AR-00-2017 & 1.1 & 7.7 & 4.9 & 11.8 & 18.8 & 18.5 & 10.1 & 4.5 & 2.8 & 1.3 & 0.8 & 0.4 & 2.2 & 0.9 & 11.4 & 2.7 \\
\hline GW1-1-2014 & 0.4 & 11.6 & 8.9 & 24.4 & 11.3 & 11.4 & 7.0 & 4.1 & 2.2 & 1.0 & 0.3 & 0.1 & 0.6 & 0.7 & 14.9 & 0.6 \\
\hline GW1-2-2014 & 0.2 & 26.0 & 9.1 & 27.6 & 5.4 & 4.8 & 1.5 & 0.5 & 0.2 & 0.1 & 0.0 & 0.0 & 0.0 & 0.3 & 23.2 & 0.5 \\
\hline GW1-3-2014 & 0.2 & 22.6 & 12.0 & 24.7 & 6.7 & 5.2 & 2.2 & 1.0 & 0.4 & 0.1 & 0.0 & 0.0 & 0.4 & 0.4 & 23.3 & 0.1 \\
\hline GW1-4-2014 & 0.2 & 18.8 & 11.4 & 24.9 & 7.1 & 6.8 & 2.9 & 1.9 & 1.2 & 0.4 & 0.1 & 0.0 & 0.6 & 0.5 & 22.4 & 0.8 \\
\hline GW1-5-2014 & 0.3 & 28.0 & 10.3 & 26.9 & 5.6 & 4.1 & 1.5 & 0.8 & 0.2 & 0.1 & 0.1 & 0.0 & 0.2 & 0.3 & 20.9 & 0.7 \\
\hline GW1-6-2014 & 0.3 & 20.5 & 13.9 & 25.5 & 6.9 & 4.8 & 2.0 & 0.9 & 0.4 & 0.0 & 0.0 & 0.0 & 0.6 & 0.4 & 22.8 & 0.5 \\
\hline GW1-7-2014 & 0.3 & 21.7 & 9.8 & 26.4 & 5.9 & 5.7 & 2.6 & 1.2 & 0.6 & 0.2 & 0.1 & 0.0 & 0.6 & 0.4 & 23.4 & 0.7 \\
\hline GW1-8-2014 & 0.2 & 26.2 & 12.7 & 22.5 & 3.7 & 2.6 & 0.8 & 0.3 & 0.0 & 0.0 & 0.0 & 0.0 & 0.4 & 0.3 & 29.2 & 0.8 \\
\hline GW1-9-2014 & 0.1 & 26.7 & 11.2 & 24.1 & 5.2 & 4.2 & 1.5 & 0.6 & 0.2 & 0.1 & 0.0 & 0.0 & 0.4 & 0.2 & 24.3 & 0.3 \\
\hline GW2-1-2015 & 0.2 & 12.7 & 13.5 & 39.1 & 9.9 & 6.9 & 2.1 & 1.0 & 0.3 & 0.0 & 0.0 & 0.0 & 0.0 & 0.3 & 13.5 & 0.2 \\
\hline GW2-2-2015 & 0.1 & 27.1 & 17.8 & 30.4 & 4.4 & 1.9 & 0.0 & 0.0 & 0.0 & 0.0 & 0.0 & 0.0 & 0.1 & 0.6 & 17.2 & 0.3 \\
\hline GW2-3-2015 & 0.4 & 5.7 & 10.8 & 48.4 & 10.1 & 4.3 & 0.1 & 0.0 & 0.0 & 0.0 & 0.0 & 0.0 & 0.0 & 0.6 & 19.2 & 0.1 \\
\hline GW2-4-2015 & 0.1 & 9.1 & 16.3 & 37.0 & 10.5 & 7.4 & 2.3 & 0.9 & 0.3 & 0.1 & 0.0 & 0.0 & 0.0 & 0.4 & 15.2 & 0.3 \\
\hline GW2-5-2015 & 0.2 & 7.4 & 7.6 & 30.2 & 15.2 & 12.4 & 5.8 & 3.1 & 1.4 & 0.4 & 0.0 & 0.0 & 0.1 & 0.3 & 15.1 & 0.4 \\
\hline GW2-6-2015 & 0.1 & 14.7 & 16.7 & 38.7 & 7.2 & 4.0 & 0.8 & 0.0 & 0.1 & 0.0 & 0.0 & 0.0 & 0.2 & 0.4 & 16.6 & 0.2 \\
\hline GW2-7-2015 & 0.2 & 6.4 & 9.4 & 27.4 & 12.7 & 8.1 & 2.9 & 1.7 & 0.9 & 0.5 & 0.1 & 0.0 & 0.3 & 0.6 & 28.0 & 0.5 \\
\hline GW2-8-2015 & 0.3 & 2.6 & 7.0 & 19.1 & 16.1 & 13.9 & 7.1 & 3.6 & 1.8 & 0.8 & 0.8 & 0.4 & 1.4 & 1.9 & 20.7 & 0.5 \\
\hline DP-25-2016 & 0.8 & 10.7 & 14.1 & 32.4 & 10.7 & 5.7 & 2.6 & 1.6 & 0.9 & 0.2 & 1.7 & 0.0 & 0.5 & 0.7 & 16.4 & 1.1 \\
\hline DP-17-2016 & 0.9 & 47.5 & 11.4 & 19.1 & 2.4 & 0.5 & 0.0 & 0.0 & 0.0 & 0.0 & 0.9 & 0.0 & 0.0 & 0.4 & 13.7 & 3.0 \\
\hline DP-24-2016 & 0.6 & 12.8 & 16.0 & 44.9 & 7.1 & 3.2 & 0.4 & 0.0 & 0.0 & 0.0 & 2.6 & 0.0 & 0.2 & 0.6 & 11.0 & 0.7 \\
\hline GW2-9-2016 & 1.2 & 15.2 & 14.9 & 38.9 & 7.9 & 3.0 & 0.5 & 0.0 & 0.0 & 0.1 & 2.6 & 0.0 & 0.2 & 0.9 & 12.8 & 1.7 \\
\hline DP-04-2016 & 0.8 & 11.4 & 15.5 & 42.9 & 6.6 & 2.9 & 0.4 & 0.0 & 0.0 & 0.0 & 2.2 & 0.0 & 0.3 & 0.7 & 15.0 & 1.3 \\
\hline GW2-3-2016 & 0.6 & 8.4 & 13.3 & 50.3 & 4.9 & 0.9 & 0.0 & 0.0 & 0.0 & 0.0 & 3.5 & 0.0 & 0.1 & 0.6 & 15.8 & 1.7 \\
\hline GW2-2-2016 & 0.2 & 51.7 & 9.4 & 19.8 & 0.0 & 0.0 & 0.0 & 0.0 & 0.0 & 0.0 & 2.5 & 0.0 & 0.0 & 0.4 & 15.5 & 0.5 \\
\hline DP-02-2017 & 0.0 & 74.6 & 1.7 & 11.0 & 0.0 & 0.0 & 0.0 & 0.0 & 0.0 & 0.0 & 1.1 & 0.0 & 0.0 & 0.1 & 11.4 & 0.2 \\
\hline
\end{tabular}




\begin{tabular}{|c|c|c|c|c|c|c|c|c|c|c|c|c|c|c|c|c|}
\hline DP-10-2017 & 0.3 & 17.3 & 8.7 & 62.6 & 4.0 & 0.4 & 0.0 & 0.0 & 0.0 & 0.0 & 3.1 & 0.0 & 0.0 & 0.2 & 3.5 & 0.0 \\
\hline DP-21-2017 & 0.5 & 27.4 & 14.8 & 41.2 & 6.3 & 2.2 & 0.1 & 0.1 & 0.0 & 0.0 & 1.4 & 0.0 & 0.1 & 0.2 & 5.6 & 0.5 \\
\hline DP-15-2017 & 0.3 & 32.7 & 19.6 & 28.3 & 1.5 & 0.1 & 0.1 & 0.0 & 0.0 & 0.0 & 1.1 & 0.0 & 0.5 & 0.2 & 15.3 & 0.5 \\
\hline DP-24-2017 & 0.6 & 26.5 & 17.8 & 38.3 & 2.1 & 0.1 & 0.1 & 0.0 & 0.0 & 0.0 & 1.3 & 0.0 & 0.5 & 0.1 & 12.3 & 0.5 \\
\hline GW2-7-2017 & 0.5 & 26.0 & 13.2 & 32.9 & 1.9 & 0.1 & 0.7 & 0.0 & 0.0 & 0.0 & 1.2 & 0.0 & 1.1 & 0.5 & 21.1 & 1.1 \\
\hline GW2-9-2017 & 0.2 & 21.7 & 12.2 & 52.4 & 0.6 & 0.0 & 0.0 & 0.0 & 0.0 & 0.0 & 2.6 & 0.0 & 0.1 & 0.3 & 9.5 & 0.4 \\
\hline GW2-3-2017 & 0.8 & 16.3 & 14.9 & 53.0 & 1.4 & 0.0 & 0.0 & 0.0 & 0.0 & 0.0 & 2.2 & 0.0 & 0.1 & 0.2 & 10.3 & 0.8 \\
\hline
\end{tabular}


Table S3. Radiocarbon data for twelve samples of acid extractable organics (AEOs) collected during the 2016 field campaign. See Table S1 for further sample information.

\begin{tabular}{|c|c|c|c|c|c|c|c|c|c|}
\hline U Ottawa ID & Sample & ${ }^{14} \mathbf{C}$ yr BP & $\mathbf{I}$ & $\mathbf{F}^{14} \mathbf{C}$ & $\mathbf{I}$ & $\mathbf{D}^{14} \mathbf{C} \%$ & $\mathbf{I}$ & $\boldsymbol{\Delta}^{14} \mathbf{C} \%$ & $\mathbf{I}$ \\
\hline \hline UOC-6325 & AR-00-2016 & $>$ Modern & --- & 1.0018 & 0.0039 & 2 & 3.9 & -6 & 3.9 \\
\hline UOC-6319 & DP-04-2016 & 9298 & 41 & 0.3143 & 0.0016 & -686 & 1.6 & -688 & 1.6 \\
\hline UOC-6320 & DP-17-2016 & 14753 & 49 & 0.1594 & 0.0010 & -841 & 1.0 & -842 & 1.0 \\
\hline UOC-6321 & DP-24-2016 & 7671 & 35 & 0.3849 & 0.0017 & -615 & 1.7 & -618 & 1.7 \\
\hline UOC-6322 & DP-25-2016 & 6958 & 40 & 0.4206 & 0.0021 & -579 & 2.1 & -583 & 2.1 \\
\hline UOC-6326 & GW2-2-2016 & 24375 & 113 & 0.0481 & 0.0007 & -952 & 0.7 & -952 & 0.7 \\
\hline UOC-6318 & GW2-3-2016 & 16839 & 69 & 0.1229 & 0.0011 & -877 & 1.1 & -878 & 1.1 \\
\hline UOC-6317 & GW2-9-2016 & 8114 & 35 & 0.3642 & 0.0016 & -636 & 1.6 & -639 & 1.6 \\
\hline UOC-6323 & MR-01-2016 & 635 & 27 & 0.924 & 0.0031 & -76 & 3.1 & -84 & 3.1 \\
\hline UOC-6324 & MR-UP-2016 & 158 & 25 & 0.9806 & 0.0030 & -19 & 3.0 & -27 & 3.0 \\
\hline UOC-6316 & SP-2016 & 27426 & 163 & 0.0329 & 0.0007 & -967 & 0.7 & -967 & 0.7 \\
\hline UOC-6315 & TP2-2016 & 35042 & 403 & 0.0127 & 0.0006 & -987 & 0.6 & -987 & 0.6 \\
\hline
\end{tabular}



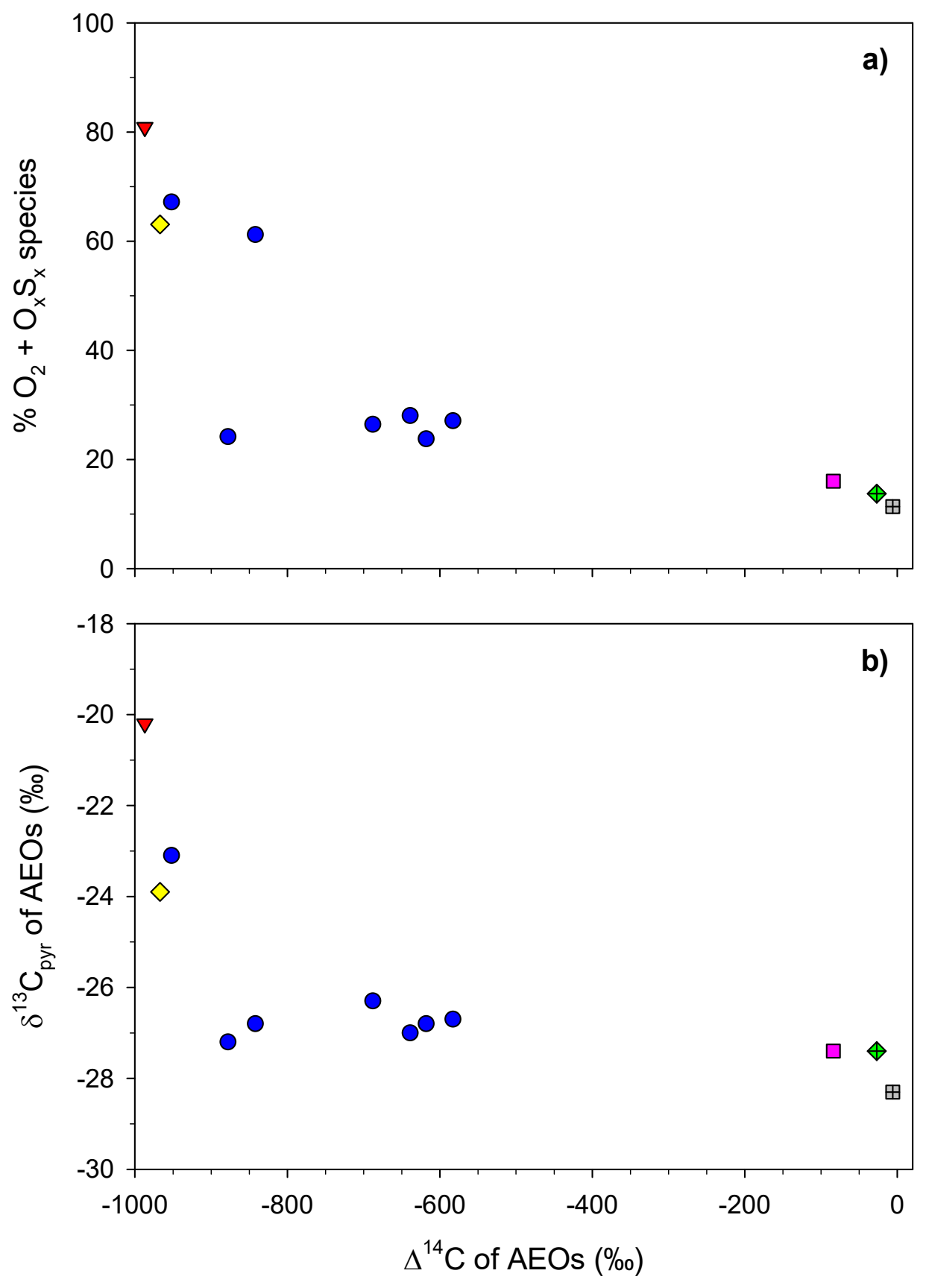

Figure S1. The relationship between a) $\Delta{ }^{14} \mathrm{C}$ and $\mathrm{O}_{2}+\mathrm{O}_{\mathrm{x}} \mathrm{S}_{\mathrm{x}}$ and b) $\Delta{ }^{14} \mathrm{C}$ and $\delta^{13} \mathrm{C}_{\mathrm{pyr}}$ in acid extractable organics (AEOs) for samples collected during the 2016 field campaign (see Table S1 for further sample details). The different symbols represent OSPW (inverted red triangle), monitoring well groundwater from Site 2 (blue circles), non-bitumen background (crossed green diamond), Athabasca River (crossed gray square), downstream Muskeg River (pink square) and seep-pipe samples (yellow diamond). 


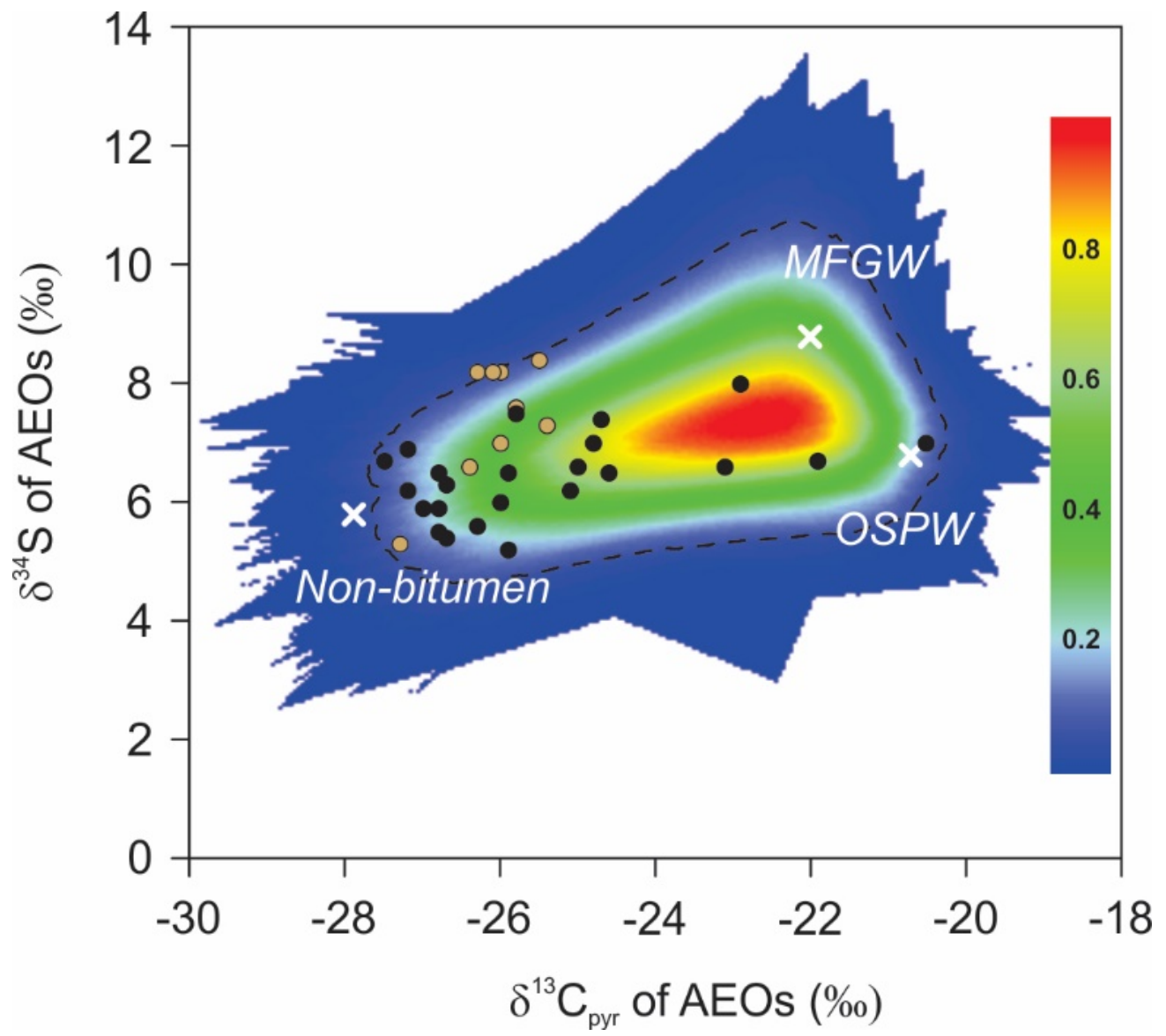

Figure S2. The simulation of all possible mixing polygons incorporating the average isotope ratios of acid extractable organics (AEOs) for the three end-members (white crosses): oil sands-processaffected water (OSPW), McMurray Formation groundwater (MFGW), and non-bitumen background. The coloured heat map illustrates the probability that a groundwater sample (gold circles, Site 1; black circles, Site 2) can be explained by the mixing model, with the dashed line representing the 5\% probability contour (Smith et al., 2013).

Smith, J.A., Mazumder, D., Suthers, I.M., Taylor, M.D., 2013. To fit or not to fit: evaluating stable isotope mixing models using simulated mixing polygons. Methods in Ecology and Evolution 4, 612-618. 